una relazione

$$
f \cdot g \cdot y=\sum_{i=1}^{m} a_{i} \cdot z_{i} \quad \text { con } a_{i} \in\left[k, x_{1}, \ldots, x_{n}\right]
$$

perchè $\left[\left(k, x_{1}\right), x_{2}, \ldots, x_{.}\right] \frown\left[\left(k, x_{2}, \ldots, x_{n}\right), x_{1}\right]=\left[k, x_{1}, \ldots, x_{n}\right]$.

Essendo quindi l'integrità di $K$ relativa a $\left[k, x_{1}, \ldots, x_{n}\right]$ un sotto- $\left.\mid k, x_{1}, \ldots, x_{n}\right]$ modulo di

$$
\sum_{i=1}^{m}\left[k, x_{1}, \ldots, x_{n}\right] \cdot \frac{z_{i}}{f \cdot g},
$$

arrà anche essa una $\left[k, x_{1}, \ldots, x\right]$-base finita (ved. 98).

\title{
CAPITOLO IV
}

\section{VARIETA}

\section{\$ 1. Varietà in generale.}

109. Se $A$ è base della prospettiva D. ogni elemento di $A$ attivo in $A$ è attivo anche in $S$ e l'oggetto di $A$ pù̀ essere identificato con quello di $S$, (identificazione, che sempre supponiamo eseguita).

Dimostraziont. - I. Sia $a \in A$ attivo in $A$ e $a \cdot x=0$ con $x \in S$. Essendo $A$ base di $\mathbb{D}$, esiste $a^{\prime} \in A$ tale che $a^{\prime} \subset \mid \mathbb{P}$ e $x \cdot a^{\prime}=a^{\prime \prime} \in A$. Allora $a \cdot a^{\prime \prime}=0$ e quindi $a^{\prime \prime}=0$, il che implica $x=0$, poichè $a^{\prime} \subset \mid=\mathbb{P}$ garantisce che $a^{\prime}$ ̀े attivo in $S$ (ved. 69).

Da cio segue che ogni elemento $\frac{a}{b}(a, b \in A, b$ attivo in $A$ ) dell'oggetto (A) di $A$ rappresenta auche un elemento dell'oggetto $(S)$ di $S$. Identificando l'elemento $\underset{b}{a}$ di $(A)$ con l'elemento $\frac{a}{b}$ di $(S)$, si definisce $(A)$ come sottoanello di $(S)$.

II. Ogni elemento $x$ di $(S)$ в quoziente $\frac{a}{b}: \frac{c}{d}$ con $a, b, c, d \in A ; b, d \subset \mathbb{D}$, $\frac{c}{d}$ attivo in $S$. Allora anche $b \cdot c=b \cdot d \cdot \frac{c}{d}$ è attivo in $S$, cosicchè esiste un elemento $y=\frac{a \cdot d}{b \cdot c}$ di $(A) \subset(S)$ soddisfacente in $(S)$ all' equazione $y \cdot \frac{c}{d}=$ $=\frac{a \cdot d \cdot c}{b \cdot c \cdot d}=\frac{a}{b}$ e percio uguale a $x$.

Con questo è dimostrato che il sottoanello $(A)$ di $(S)$ riempie tutto l'og. getto $(S)$.

110. Defrnizione. - Una varietà $̇$ an insieme di prospettive (oppure di aspetti) soddisfacente alle condizioni seguenti: 
I) Due prospettive qualunque appartenenti ad una medesima varietà hanno base comune.

II) Se l'aspetto s appartiene ad una varietà, ogni prospettiva di base $s$ appartiene altresì a questa varietò.

Stabiliamo ohe il solo soddisfare a queste condizioni qualifica un insieme di prospettive come varietà.

Dalla prima proprietà delle varietà segue subito, tenuto conto del teorema 109:

Tutte le prospettive costituenti una varietà sono prospettive di un medesimo oggetto

che chiameremo l'oggetto della varietà, mentre la varietà stessa sarà detta semplicemente u n a varietà del suo oggetto. Diciano «una», perchè uno stesso oggetto ammette in generale una infinità di varietà.

Una varietà sarà chiamata prima, primaria, non-primaria secondochè il suo oggetto sia corpo, oggetto primario; anello non-primario.

111. Due prospettive appartenenti ad una stessa varietà non ammettono mai una estensione comune.

Dimostrazione. - Se $\mathbb{P}$ è estensione tanto di $\mathfrak{p}_{1}$ quanto di $\mathfrak{p}_{2}$, valgono le relazioni $\mathbb{P} \cap s_{1}=\mathfrak{p}_{1}, \mathbb{P} \cap s_{2}=\mathfrak{p}_{2}, s \supset s_{1}, S \supset s_{2}$ che dànno

$$
\mathfrak{p}_{1} \frown s_{1} \frown s_{2}=\mathbb{D} \frown s_{1} \frown s_{2}=\mathfrak{p}_{2} \frown s_{1} \frown s_{2} .
$$

Supposto che $\mathfrak{p}_{1}, \mathfrak{p}_{2}$ appartengano ad una stessa varietà, esse avranno base comane per la quale sempre può scegliersi l'intersezione $s_{1} \frown s_{2}$. Le relazioni suddette mostrano allora, in quanto esprimono la coincidenza degli ideali che individuano $\mathfrak{p}_{1}, \mathfrak{p}_{2}$ in $s_{1} \frown s_{2}$, che si tratta di una sola prospettiva $p_{1}=p_{2}$.

112. Per due prospettive $\mathfrak{p}_{1}, \mathfrak{p}_{2}$ appartenenti ad una stessa varietà, da $\mathfrak{p}_{2} \frown s_{1} \subset \mathfrak{p}_{2}$ segue sempre $s_{1} \subset s_{2}$ e viceversa.

Dimostrazione. - Ogni $x \in s_{1}$ è quoziente $\frac{a}{b}$ con $a, b \in s_{1} \frown s_{2}, b \subset \mid=\mathfrak{p}_{1}$ (ved. 110 I). Da $\mathfrak{p}_{2} \cap s_{1} \subset \mathfrak{p}_{1}$ segue che $b$ non appartiene nemmeno $a p_{2} \frown s_{1}$ e che perciò $\frac{a}{b}$ e anche un elemento di $s_{2}$.

La proposizione reciproca, ciò̀ il fatto che da $s_{1} \subset s_{2}$ segua $\mathfrak{p}_{2} \cap s_{1} \subset \mathfrak{p}_{1}$, è conseguenza del teorema 65 , non potendosi verificare il caso $s_{1} \subset \mathfrak{p}_{2}$, perchè allora l'elemento 1 di $s_{1} \subset s_{2}$, che coincide con l'elemento 1 di $s_{2}$, (essendo questo l' elemento 1 dell'oggetto della varieta), sarebbe contenuto in $\mathbf{p}_{2}$. 
E utile, a proposito del teorema or ora dimostrato, rappresentare gli nspetti e le origini corrispondenti, che in certo senso sono gli orizzonti delle prospettive, come se si trattasse di campi e dei loro contorni:

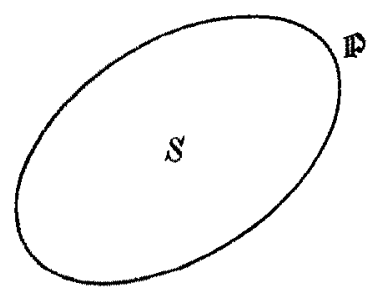

Il teorema suddetto dice allora che nel caso di prospettive appartenenti alla stessa varietà si hanno necessariamente le situazioni:
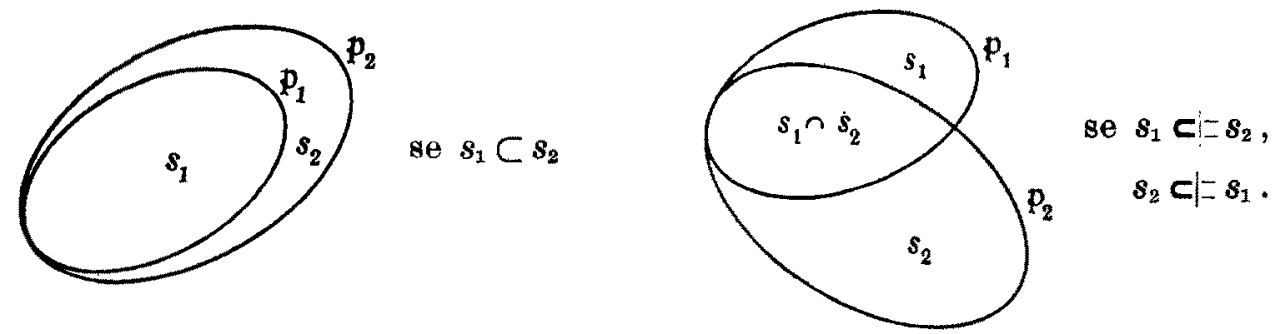

113. Se $M$ designa un insieme di prospettive, semplifichiamo la locu. zione dicendo $s \in M$ invece di dire che la prospettiva presentante $s$ appartiene a $M$.

Ogni insieme $M$ di prospettive, tale che due qualunque fra le prospettive di $M$ abbiano base comune, diventa varietà aggiungendovi tutte le prospettive che hanno come base un aspetto appartenente a $M$.

Dimosmazione. - Siano $\mathbb{D}_{1}, \mathbb{P}_{2}$ prospettive qualunque aventi come basi gli aspetti $s_{1}$, risp. $s_{2}$ appartenenti a $M$. Poiche $\mathfrak{p}_{1}, \mathfrak{p}_{2}$ hanno base comune $A \subset s_{1} \cap s_{2}$, ogni elemento di $s_{1}$ è quoziente $\frac{a}{b}$ con $a, b \in s_{1} \cap s_{2}, b \subset \mid=\mathfrak{p}_{1}$. Ma essendo $s_{1}$ base di $\mathbb{P}_{1}$ e pertanto $\mathbb{p}_{1} \frown s_{1} \neq s_{1}$, si ha $\mathfrak{p}_{1} \supset \mathbb{P}_{1} \frown s_{1}$ e quindi $b c \mid=D_{1}$. Sono verificate danque le premesse del teorema 75 da cui segue che $s_{1} \frown s_{2}$ \& base di $\mathbb{P}_{1}$ (e per ragione simile di $\mathbb{P}_{2}$ ).

$L$ 'insieme $M^{\prime}$ ottenuto da $M$ nella maniera suddetta ha dunque la proprietà I caratteristica per le varietà.

Sia ora $D_{2}$ una prospettiva qualunque arente come base un aspetto $S_{1} \in M^{\prime}$. Esiste allora nn aspetto $s \in M$, base di $\mathbb{D}_{1}$. Ancor qui valgono le 
premesse del teorema 75, essendo ogni elemento di $s_{1}$ quoziente $\frac{a}{b}$ con $a, b \in s$, $b \subset \mid=\mathbb{P}_{1} \supset \mathbb{P}_{2} \frown S_{1} \supset \mathbb{P}_{2} \frown s$, ciò̀ $b \subset \mid=\mathbb{P}_{2}$. Ne concludiamo che $s \in M$ è base anche della prospettiva $\mathbb{P}_{2}$, cosicchè questa appartiene all'insieme $M^{\prime}$.

Verificata anche la seconda proprietà caratteristica delle varietà, ¿̀ dimostrato il teorema.

114. Ogni anello A diverso dall' ideale generato dai suoi elementi inattivi $\grave{e}$ base di una varietà, e ciò̀ della totalità $V(A)$ delle prospettive di base $A$.

Dimostrazrone. - L'ipotesi che l'ideale a generato da tutti gli elementi inattivi di $A$ sia $\neq A$ garantisce l'esistenza di un ideale primo $c \neq A$ conte. nente tutti gli elementi inattivi di $A, \Theta$ questo $c$ individua (ved. 72) una prospettiva di base $A$.

I. Dae qualunque prospettive appartenenti a $V(A)$ hanno la base comune $A$.

II. Ogni prospettiva $\mathbb{D}_{2}$ avente come base un aspetto $S_{1} \in V(A)$ ha altresi la base $A$ (ved. 75 ), essendo ogni elemento di $S_{1}$ quoziente $\frac{a}{b}$ con $a, b \in A, b \subset \mid=\mathbb{P}_{1} \supset \mathbb{P}_{2} \cap S_{1} \supset \mathbb{P}_{2} \cap A$, cioè $b \subset \mid=\mathbb{P}_{2}$, il che mostra $S_{2} \in V(A)$. c. d. d.

\section{§ 2. La sintesi di varietà.}

115. Per preparare la nozione di sintesi di varieta ci vuole qualche osservazione sull' attività di anelli.

Se $A_{0}$ è sottoanello di $A$, può ben darsi che un elemento di $A_{0}$ attiro in $A_{0}$ sia inattivo in $A$.

Chiameremo un anello $A_{0}$ attivo in $A$ se $A_{0}$ è un sottoanello di $A$ avente almeno un elemento attivo in $A_{0} e$ ogni elemento di $A_{0}$ attivo in $A_{0}$ e attivo anche in $A$. E chiaro che ogni elemento attivo in un anello $\dot{e}$ attivo anche nell'oggetto di questo.

Nel caso di un anello $A_{0}$ attivo in $A$ s'identifichi sempre l'elemento $\frac{a}{b}\left(a, b \in A_{0}, b\right.$ attivo in $\left.A_{0}\right)$ dell'oggetto $\left(A_{0}\right)$ con l'elemento $\frac{a}{b}$ dell'oggetto $(A)$. Possiamo dire allora:

$L$ 'oggetto di un anello attivo in $A$ è attivo nell'oggetto di $A$.

Invero, da $\frac{a}{b} \cdot \frac{x}{y}=0(x, y \in A, y$ attivo in $A)$ segue $a \cdot x=0$ e quindi $x=0$, ciò $\frac{x}{y}=0$, se $\frac{a}{b}$ è attivo in $\left(A_{0}\right)$, cioè $a$ attivo in $A_{0}$ e quindi in $A$. 